\title{
Inhibition of methicillin resistant Staphylococcus aureus by a plasma needle
}

Research Article

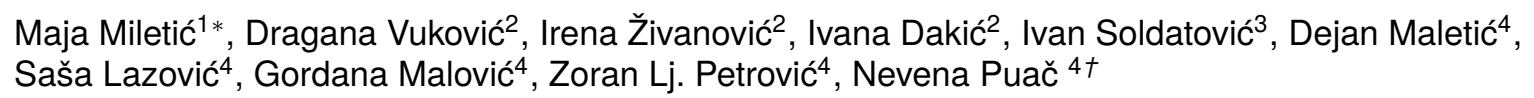

1 Faculty of Dental Medicine,

University of Belgrade, dr Subotica 8, 11000 Belgrade, Serbia

2 Institute of Microbiology and Immunology,

Faculty of Medicine, University of Belgrade, dr Subotica 1, 11000 Belgrade, Serbia

3 Institute for Medical Statistics and Informatics,

Faculty of Medicine, University of Belgrade, dr Subotica 1, 11000 Belgrade, Serbia

4 Institute of Physics,

University of Belgrade, Pregrevica 118, 11080 Belgrade, Serbia

Received 19 July 2013; accepted 30 December 2013

\begin{abstract}
:
In numerous recent papers plasma chemistry of non equilibrium plasma sources operating at atmospheric pressure has been linked to plasma medical effects including sterilization. In this paper we present a study of the effectiveness of an atmospheric pressure plasma source, known as plasma needle, in inhibition of the growth of biofilm produced by methicillin resistant Staphylococcus aureus (MRSA). Even at the lowest powers the biofilms formed by inoculi of MRSA of $10^{4}$ and $10^{5} \mathrm{CFU}$ have been strongly affected by plasma and growth in biofilms was inhibited. The eradication of the already formed biofilm was not achieved and it is required to go to more effective sources.
\end{abstract}

PACS (2008): 52.80.Pi, 87.18.Fx, 92.20.jb

Keywords: $\quad$ non-thermal atmospheric plasmas $\cdot$ plasma needle $\cdot$ antimicrobial $\cdot$ methicillin resistant Staphylococcus aureus $\cdot$ biofilm

(c) Versita sp. z o.o.

\section{Introduction}

The majority of bacteria in nature have a tendency to interact and grow in close association with surfaces, form-

*E-mail: maja.miletic@stomf.bg.ac.rs (Corresponding author)

†E-mail:nevena@ipb.ac.rs ing biofilms. A biofilm can be defined as a surfaceattached community of bacteria growing embedded in a self-produced matrix composed of extracellular polymeric substances (EPS) [1,2]. Bacteria within biofilms have metabolic and physiological capabilities which are not associated with individual, unattached cells. Notable amongst these unique properties is high level of resistance to antibiotics and chemical/physical decontamination procedures. Therefore, bacteria living in biofilms are 
difficult or even impossible to eradicate $[1,2]$. The aim of this investigation was to evaluate the antimicrobial activity of a non-thermal atmospheric plasma, generated by a specifically designed plasma needle device, against biofilms produced by methicillin resistant Staphylococcus aureus (MRSA). Recently atmospheric pressure discharges have been developed as sources of low temperature plasmas at atmospheric pressure and, thus, it became possible to develop plasma medical applications $[3,4]$. Most studies have shown very good results [5-8] in direct contact of plasmas with microorganisms. However sterilization of planktonic samples and biofilms proved to be more difficult [9-13]. In this paper we extend the application of a plasma needle that has been tested in direct contact with plant and mammalian cells $[14,15]$ and for planktonic samples of bacteria [15] to study sterilization of biofilms. It is estimated that up to $80 \%$ of human bacterial infections are actually biofilm-associated. Biofilm formation has typically been implicated in persistent tissue infections and medical device-related infections [16], although the role of biofilm has recently been recognized in acute infections as well [17]. Species of the genus Staphylococcus, in particular S. aureus and S. epidermidis, are among the most frequent causative agents of biofilm-mediated infections $[18,19]$. The most important $S$. aureus diseases that have a demonstrated biofilm component are osteomyelitis, medical device-related infections, wound infections and endocarditis. Worldwide dissemination of MRSA strains that display resistance to all beta-lactam antibiotics, further complicates prevention and treatment of these diseases $[18,19]$. Since antibiotic treatment often fails to overcome biofilm-associated infections, particularly those caused by multidrug resistant bacteria, it is apparent that development of alternative strategies for preventing and/or treating these infections is of great importance. Further research is needed to understand molecular mechanisms of biofilm formation by bacteria. For example, it has recently been shown that S. aureus cysteine proteases ScpA and SspB, so called Staphopains, are the key modulators of biofilm production by this bacterium and that development of strategies to up-regulate the Staphopains could be a novel approach to treating $S$. aureus biofilm infections [20]. Lately, it was shown that low-temperature gas plasma presents a powerful medical tool in general $[3,4,21]$. Cold plasma can successfully eradicate microorganisms [22-24] and this fact is exploited in plasma sterilization of medical equipment and instruments $[25,26]$. Compared to common methods used in health-care facilities like steam under pressure, dry heat, ethylene oxide gas and liquid chemicals, plasma offers non-toxicity and treatment of heat sensitive instruments without rapid degradation. Plasmas can offer dif- ferent principles of inactivation mechanisms like etching or sputtering of membranes of bacteria or endospore coat, charging of the bacterial cell membrane, DNA modifications based on strand breaks [27]. For all inactivation mechanisms maximum result is obtained through synergistic effects of all plasma agents (electrons, ions, photons, electric fields, radicals and metastables) [28] that target many cellular components and metabolic processes in bacteria. This explains one of the major advantages of cold atmospheric plasmas i.e. multiple targets in bacterial cells make the emergence of resistance mechanisms less likely [9]. In addition, plasma chemistry is important as generation of radicals or active molecules at the surface in very small quantities may trigger a biological response with only a small amount of reactive species that would otherwise be very toxic. The effectiveness of the sterilization process depends not only on plasma composition, but also on intrinsic properties of the sample. For example, individual bacteria are easily inactivated by UV radiation at a timescale of seconds [29]. On the other hand, only UV radiation is not sufficient when bacteria are contained in some surrounding medium, especially if this medium has a supporting structure. Good examples are bacteria in suspensions and bioflims [30]. Our previous work showed plasma sterilization of bacteria in planktonic sample despite the obvious "shielding" by the liquid medium [15]. Independent of our work, Joshi et al. reached similar conclusions [13]. A large number of papers followed [31-33]. In this sense, biofilms represent an even more challenging task because bacteria are shielded by the polymeric matrix. Plasma cannot easily penetrate complex porous and hollow structures and, in addition, a synergetic effect of agents is needed for biomaterial removal and indepth effects on multilayer stacks. As we move towards the in-vivo applications, the situation gets more complicated due to the presence of biofilms containing several species of bacteria, blood, fat, and other body products like sweat or saliva. Despite the problems, application of lowtemperature plasmas at atmospheric pressure presents a promising antibiofilm approach according to the literature.

\section{Experimental}

\subsection{Bacterial strain and growth conditions}

The MRSA isolate used in this study was recovered from a surgical wound and identified as MRSA by BD Phoenix Automated Microbiology System (Becton Dickinson Diagnostic Systems, Sparks, MD). Identification to the species level was confirmed by detection of the nuc gene [34] whilst resistance to meticillin was established by polymerase chain reaction (PCR) for the mecA gene [35]. Pre- 
vious evaluation identified the strain as a strong biofilm producer [36]. The MRSA strain was transferred from a frozen stock culture onto tryptic soy agar and incubated aerobically for 24 hours at $35-37^{\circ} \mathrm{C}$. The bacterial suspensions were prepared as follows: a few colonies were suspended in physiological saline and the turbidity of the suspension was adjusted to $0.5 \mathrm{McF}$ arland stan-

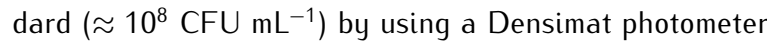
(BioMerieux, France). The final testing inocula used in this study ranged from $10^{6}$ to $10^{4} \mathrm{CFU}$ per well of a sterile 96 well-flat bottom polystyrene microtiter plate.

\subsection{Plasma source}

In this study we used a plasma needle device (developed in our laboratory at the Institute of Physics of the University of Belgrade) that was designed for biomedical applications and tested with numerous diagnostic procedures [14, 37]. The device is similar in concept to the one developed by Stoffels et al. $[38,39]$. The plasma needle consists of a central wolfram electrode $(0.5 \mathrm{~mm}$ in diameter) covered by a ceramic tube. The ceramic tube serves to insulate the central electrode from the working gas [14]. The powered electrode and the ceramic tube are placed in a glass tube with a $4 \mathrm{~mm}$ inner and $6 \mathrm{~mm}$ outer diameter. Helium is flowing between the ceramic and the glass tube and allows plasma formation only at the tip of the electrode. The body of the plasma needle is made of Teflon. Plasma needle operates at $13.56 \mathrm{MHz}$ with the electrical circuit consisting of a signal generator, amplifier and matching network.

Electrical characterization of the system is performed by using derivative probes. The probes are placed as close as possible to the tip of the needle in order to obtain the actual power transmitted to the plasma. An oscilloscope and a computer are used to capture and process the signals. The collected signals are transferred to the frequency domain by using Fast Fourier transform. In this domain current and voltage signals are corrected according to calibration curves. Conversion back to the time domain by inverse fast Fourier transform is carried out in the final stage. The difference between signals when plasma is lit and without the plasma (no helium flow) contains the information about the power transferred to the plasma. Knowledge of power transmitted to the plasma gives us a good control of treatment conditions.

\subsection{Biofilm growth}

Microtiter biofilm assay was carried out in accordance with the protocol described by Stepanović et al. [40]. Each well contained $180 \mu \mathrm{L}$ of brain heart infusion broth (BHI) supplemented with $1 \%$ glucose and $20 \mu \mathrm{L}$ of bacterial suspension. The negative control wells contained $200 \mu \mathrm{L} \mathrm{BHI}$ supplemented with $1 \%$ glucose, only. In order to assess the inhibitory effects of non-thermal plasma on growth of MRSA biofilm, the bacteria were exposed to the plasma $5 \mathrm{~h}$ after the addition of bacterial suspension to medium. After plasma treatment the biofilms were allowed to grow for $24 \mathrm{~h}$ at $35-37^{\circ} \mathrm{C}$ and then quantified. To evaluate possible effects of non-thermal plasma on the formed MRSA biofilm, the inoculated plates were first incubated for $24 \mathrm{~h}$ at $35-37^{\circ} \mathrm{C}$ and then exposed to plasma treatment. Quantification of the biofilm was performed after the plasma treatment.

\subsection{Plasma treatment conditions}

The same set of conditions was applied both to freshly inoculated plates and plates with already developed biofilms. The plasma needle was placed vertically above the microtiter plate in line with the upper edge of each well (Figure 1).

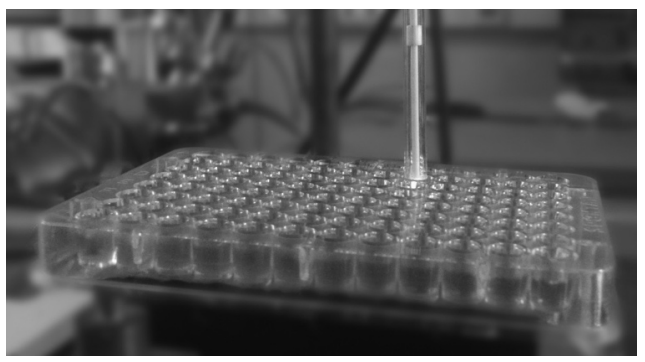

Figure 1. Plasma needle with microtiter plate.

The distance between the tip of the plasma needle and the surface of the sample in each well was fixed to $3 \mathrm{~mm}$. The samples were treated by plasma operating at three different powers $(0.15,0.9$ and $1.6 \mathrm{~W})$, and two flows of helium $(0.5$ and $1 \mathrm{~s} / \mathrm{m})$ during three exposure times (30, 60 and $120 \mathrm{~s}$ ). The untreated wells were used as positive controls. All treatments were performed in triplicate and repeated at least two times for each bacterial inoculum tested.

\subsection{Quantification of biofilm}

The content of a microplate was poured off and each well was washed three times with $300 \mu \mathrm{L}$ of sterile phosphatebuffered saline (PBS; $\mathrm{pH}$ 7.2) to remove free floating bacteria. After fixation of adherent biofilm with $150 \mu \mathrm{L}$ of methanol per well for $20 \mathrm{~min}$, the plates were emptied by flicking and left to air dry overnight in an inverted position at room temperature. The biofilms were 
stained with $150 \mu \mathrm{L}$ of $2 \%$ crystal violet for $15 \mathrm{~min}$. Excess stain was washed under running tap water. After the plates were air dried, the dye bound to the adherent cells was resolubilized with $150 \mu \mathrm{L}$ of $95 \%$ ethanol. The optical density (OD) of each well was measured at $570 \mathrm{~nm}$ using Multiskan EX reader (Labsystems). The results obtained were averaged and expressed as numbers. The cut-off OD (ODc) was defined as three standard deviations (SD) above the mean OD of the negative control [40]. The results were classified as follows: $\mathrm{OD} \leq \mathrm{ODc}$ no biofilm production; $\mathrm{OD} c \leq \mathrm{OD} \leq 2 \times \mathrm{ODc}$ weak biofilm production; $2 x \mathrm{OD} c \leq \mathrm{OD} \leq 4 \times \mathrm{ODc}$ moderate biofilm production; 4xODc $\leq$ OD strong biofilm production. Statistical analysis (One-way ANOVA, Dunett test) was performed using SPSS statistical software package (SPSS 15.0 (Chicago, Illinois)). Statistical significance was declared as $p<0.05$.

\section{Results}

The study evaluated efficacy of the low-temperature plasma at atmospheric pressure generated by an in-house designed plasma needle device against biofilm formation by a MRSA strain. Summarized results of the microtiter biofilm assays are expressed as qualitative categories in Table 1.

Table 1. Qualitative evaluation of efficacy of low-temperature plasma against biofilm formation by MRSA . Notations: No biofilm formation (0); Weak biofilm formation (+); Moderate biofilm formation $(++)$; Strong biofilm formation $(+++)$; Flow 1 (flow rate of $\mathrm{He}$ of $0.5 \mathrm{slm}$ ); Flow 2 (flow rate of $\mathrm{He}$ of $1 \mathrm{slm}$ ).

\begin{tabular}{cccccccc}
\hline Power [W] & $\begin{array}{c}\text { Exposure } \\
\text { time }[\mathrm{s}]\end{array}$ & $10^{4}$ & CFU & $10^{5}$ & CFU & $10^{6}$ & $C F U$ \\
\hline \hline \multirow{6}{*}{0.15} & 30 & ++ & + & ++ & ++ & ++ & ++ \\
& 60 & + & + & ++ & + & ++ & + \\
& 120 & ++ & + & + & + & + & + \\
\hline \multirow{4}{*}{0.9} & 30 & + & + & + & + & ++ & ++ \\
& 60 & + & + & + & + & + & + \\
& 120 & 0 & 0 & 0 & 0 & + & + \\
\hline \multirow{4}{*}{1.6} & 30 & 0 & 0 & + & + & + & + \\
& 60 & 0 & 0 & 0 & 0 & + & + \\
& 120 & 0 & 0 & 0 & 0 & + & 0 \\
\hline
\end{tabular}

The experimental design included four major variables: three different bacterial inocula were exposed to three different powers of plasma and two flows of helium during three exposure times.

It is obvious that the powers of $0.9 \mathrm{~W}$ and $1.6 \mathrm{~W}$ achieved a complete inhibition of biofilm growth for testing inocula of
$10^{4}$ and $10^{5} \mathrm{CFU}$. The highest power, 1.6 W, exhibited this effect irrespective of the treatment time, while the power of $0.9 \mathrm{~W}$ completely prevented biofilm formation only after the longest exposure of $120 \mathrm{~s}$. On the other hand, total inhibition of biofilm production by $10^{6} \mathrm{CFU}$ required maximal plasma parameters, namely power of $1.6 \mathrm{~W}$, flow rate of $1 \mathrm{slm}$ and exposure for $120 \mathrm{~s}$.

In order to quantitatively assess the antibiofilm activity of the non-thermal atmospheric plasma, the mean OD values of treated samples were compared to those of untreated controls. The mean OD values with standard deviations obtained at the flow rates of $0.5 \mathrm{slm}$ and $1 \mathrm{slm}$ are shown in Figures 2 and 3, respectively. All OD values equal to or lower than ODc values shown in Figures 2 and 3 indicate absence of biofilm since ODc was defined as three SDs above the mean $\mathrm{OD}$ of the negative control, i.e. wells containing growth medium only.

Combination of lower flow rate and minimal plasma power of $0.15 \mathrm{~W}$ was ineffective for all inocula and exposure times (Fig. 2). Higher plasma powers (0.9 W, 1.6 W) significantly decreased biofilm production by $10^{4} \mathrm{MRSA}$ cells regardless of the exposure time (Fig. 2A), while a decrease in biofilm production by $10^{5}$ MRSA cells required a longer exposure, i.e. 60 and $120 \mathrm{~s}$ (Fig. 2B). The biofilm production by the highest bacterial inoculum was not affected by the plasma treatments at the flow rate of $0.5 \mathrm{slm}$ (Fig. 2C). At the flow rate of $1 \mathrm{slm}$, plasma significantly reduced biofilm formation in samples with $10^{4} \mathrm{CFU}$, even when the smallest power of plasma was applied, during all treatment times (Fig. 3A). As far as larger inocula are concerned, $10^{5}$ and $10^{6} \mathrm{CFU}$, higher plasma powers of 0.9 and $1.6 \mathrm{~W}$ and exposure time of at least $60 \mathrm{~s}$ were needed for significant reduction in biofilm growth (Fig. 3B and 3C). The effects of non-thermal plasma on formed MRSA biofilm were evaluated by using the same set of plasma treatments. No significant reduction in biofilm was noted, even with maximal plasma parameters applied (data not shown).

\section{Discussion}

The use of atmospheric pressure non-thermal plasma has been evaluated for many potential biomedical applications, including eradication of microorganisms $[8,11]$. The cold plasma has been proven to be effective in terms of microbial inactivation and surface decontamination and sterilization [41, 42]. Numerous studies showed activity of non-thermal plasma against different Gram-negative and Gram-positive bacteria [13, 15, 43-46]. However, data on activity of cold atmospheric pressure plasma for eradication of biofilm produced by MRSA are still lim- 

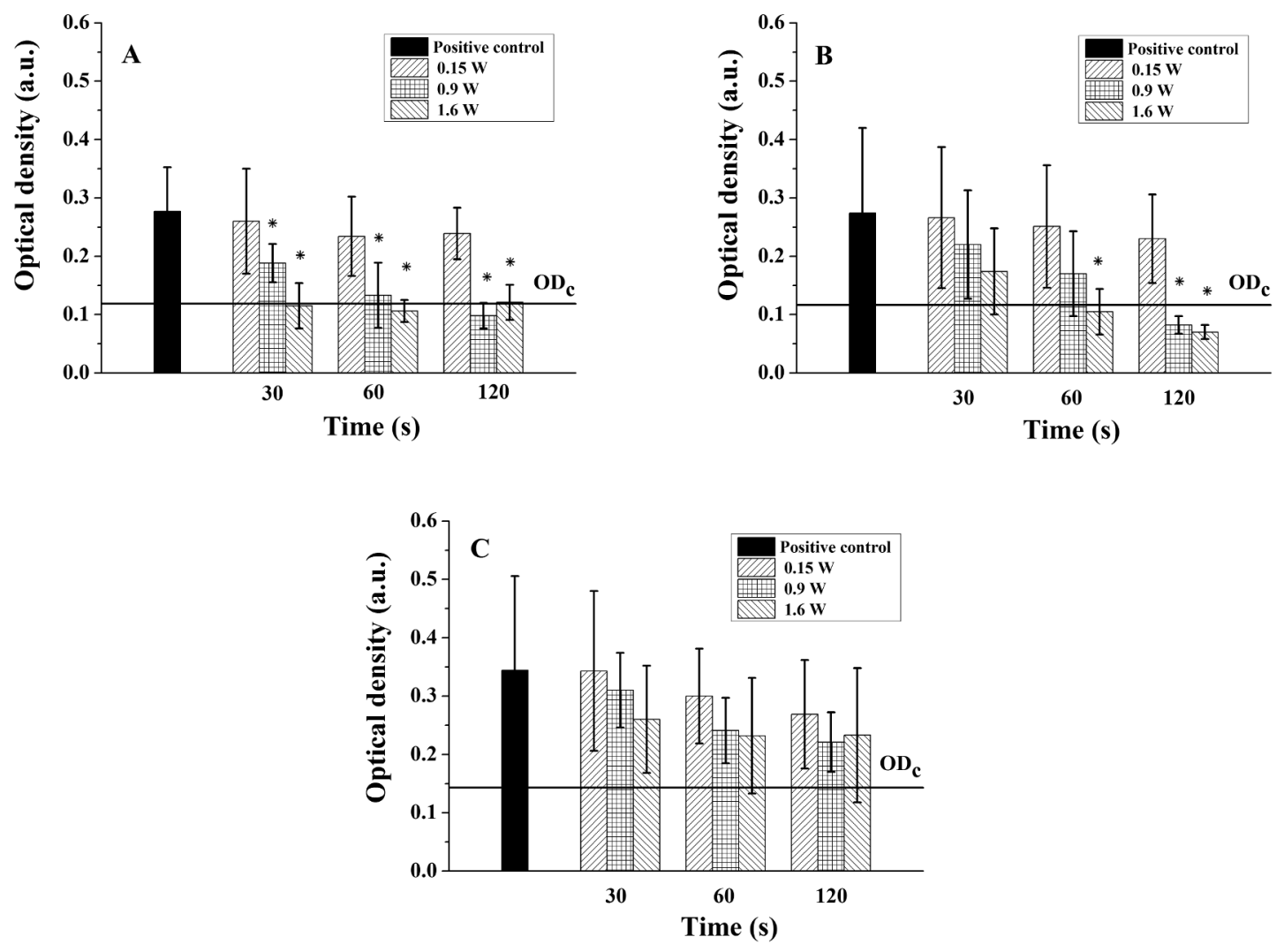

Figure 2. Efect of non-thermal atmospheric plasma on MRSA biofilm for helium flow of $0.5 \mathrm{~s} / \mathrm{m}$. Samples with different inoculum size of MRSA ((A) $10^{4} \mathrm{CFU;} \mathrm{(B)} 10^{5} \mathrm{CFU}$; (C) $\left.10^{6} \mathrm{CFU}\right)$ were treated for three plasma powers $(0.15 \mathrm{~W}, 0.9 \mathrm{~W}, 1.6 \mathrm{~W})$ and three exposure times $(30 \mathrm{~s}$, $60 \mathrm{~s}, 120 \mathrm{~s}$ ). Control samples were untreated cells. The results are presented as mean OD values of triplicates $\pm S D$ of two separate experiments. ODc was defined as 3 SDs above the mean OD of the negative control. $* p<0.05$ compared to untreated, control cells (Dunett test).

ited $[13,47]$. MRSA strains are among the leading causes of healthcare-associated infections and are a major concern for infection control programs. It is also generally appreciated that the staphylococci, including MRSA, have the ability to adhere to many types of surfaces and develop biofilms. MRSA biofilms, in addition to the multiresistance of the bacterium, have innate resistance to antimicrobial agents, and, thus, new treatment strategies that target MRSA biofilm are needed. We carried out a comprehensive in vitro investigation of the activity of atmospheric pressure non-thermal plasma generated by an in-house designed plasma needle against biofilm produced by a clinically relevant MRSA strain. Assessment of a particular plasma source is important since the working parameters for the application of different lowtemperature sources of the atmospheric plasma are difficult to be standardized, and are defined separately for every single source.
The MRSA cell densities varied from $10^{4}$ to $10^{6}$ per well and the effects of changing the plasma parameters such as power and flow rate at three different exposure times were evaluated. The results obtained clearly show that the low temperature atmospheric pressure plasma generated by a plasma needle exhibited inhibitory effects against MRSA biofilm growth. In general, inhibitory effects of the plasma tested were positively correlated to the plasma parameters. We found that effectiveness of the plasma was time and power dependent and that enhanced anti-biofilm activity was also accomplished by a higher helium gas flow. Namely, with a higher flow rate more reactive plasma agents come in contact with bacterial cells. The higher power delivered to the plasma means that concentration of the radicals and ions is increased compared to the lower powers while still staying well within the limits of negligible temperature change. As a plasma needle produces negligible amount of ozone, in this case the most important radical is $\mathrm{NO}$ which is 

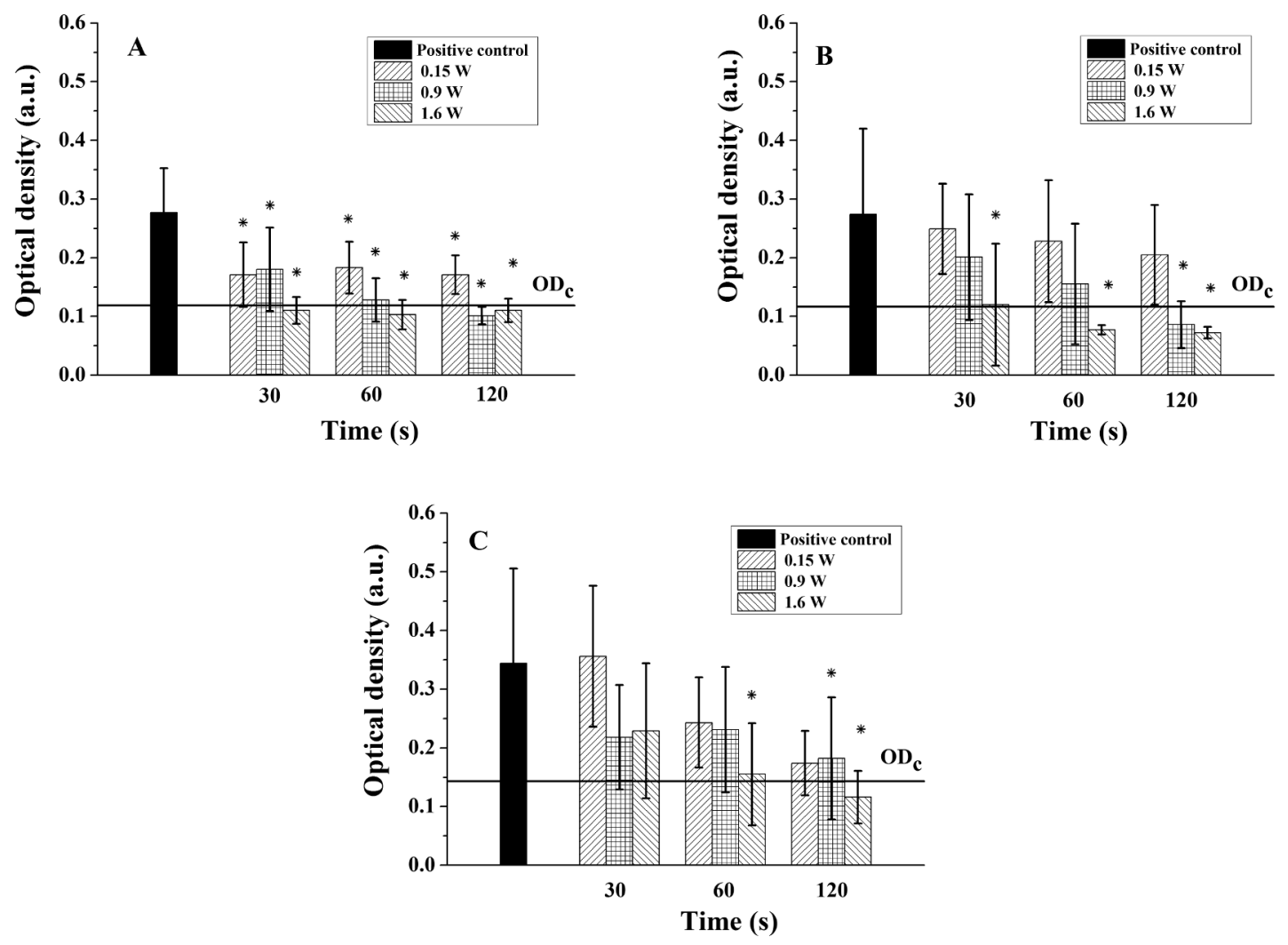

Figure 3. Efect of non-thermal plasma on MRSA biofilm for helium flow of $1 \mathrm{slm}$. Samples with different inoculum of MRSA ((A) $10^{4} \mathrm{CFU;} \mathrm{(B)} 10^{5}$ $\mathrm{CFU}$; (C) $\left.10^{6} \mathrm{CFU}\right)$ were treated for three plasma powers $(0.15 \mathrm{~W}, 0.9 \mathrm{~W}, 1.6 \mathrm{~W})$ and three exposure times $(30 \mathrm{~s}, 60 \mathrm{~s}, 120 \mathrm{~s})$. Control samples were untreated cells. The results are presented as mean OD values of triplicates $\pm S D$ of two separate experiments. ODc was defined as 3 SDs above the mean OD of the negative control. $* p<0.05$ compared to untreated, control cells (Dunett test).

a potent antimicrobial agent, effective against a range of Gram-negative and Gram-positive bacteria, including $S$. aureus [48]. In our previous work we have shown that plasma needle generates $\mathrm{NO}$ radicals and that amount of generated NO is highly dependent on the power delivered to the plasma [37]. It was shown that with an increase in power delivered to the plasma concentration of $\mathrm{NO}$ increases. As another point we have to mention the abundance of ions created by a plasma needle whose concentration also increases with power. An increase in power means a larger plasma volume and, thus, larger area is in direct contact with the plasma. The exact composition of bactericidal agents produced by plasma varies depending on geometry, composition of working gas, humidity, power, etc. The antimicrobial properties of NO may be elicited by direct modification of biomacromolecules or by formation of reactive nitrogen oxide species (RNOS) such as peroxynitrite $\left(\mathrm{OONO}^{-}\right)$, S-nitrosothiols (RSNO), nitrogen dioxide $\left(\mathrm{NO}_{2}\right)$, dinitrogen trioxide $\left(\mathrm{N}_{2} \mathrm{O}_{3}\right)$, and dinitrogen tetroxide $\left(\mathrm{N}_{2} \mathrm{O}_{4}\right)$. These reactive intermediates ex- ert antimicrobial effects by inducing lipid peroxidation or altering DNA according to Schairer et al. [49] RNOS can cause nitrosation of protein thiols and the nitrosylation of metal centres (Fe-S), ultimately modifying the functions of proteins that are essential to cellular processes $[50,51]$.

The results of the present study show that biofilm inhibition by the plasma, in addition to the plasma parameters, was also closely dependent on the inoculum size. For the largest inoculum of bacteria, plasma treatment did not affect biofilm formation at the flow rate of $0.5 \mathrm{slm}$, even with the maximum power and the longest exposure. Inhibitory effects of the plasma against biofilm produced by $10^{6}$ bacteria were observed only at the flow rate of $1 \mathrm{slm}$, in combination with other plasma parameters. Consistently high level of biofilm inhibition obtained for the smallest inoculum of $10^{4}$ MRSA cells was not sensitive to the changes in treatment times. At the flow rate of $0.5 \mathrm{slm}$, plasma power of $0.9 \mathrm{~W}$ was sufficient to decrease significantly biofilm formation by this inoculum, irrespective of the exposure 
time. At the higher flow rate, all combinations of plasma parameters were effective in biofilm growth inhibition.

While the inhibitory effects of the cold plasma on biofilm formation by the MRSA strain tested in the present study were apparent, the plasma treatments of formed biofilms, i.e. biofilms grown after 24 hours of incubation were ineffective. This may be related to the thickness of the biofilm and the inability of bactericidal agents produced by plasma to penetrate a thick biofilm. Since eradication of biofilm is essential for possible practical application of the nonthermal atmospheric plasma, further evaluation of its effects on biofilm formed after $24 \mathrm{~h}$ as well as longer incubation periods is needed. In addition to the biofilm incubation period, all parameters of the plasma treatment should be optimized in the context of biofilm eradication. It opens a possibility of further tests depending on the targeted substrates which would include extended period and repeated treatments, higher powers, a different more energetic plasma (perhaps for non living substrates) and combined treatment with other techniques. It is noteworthy that we have shown that even with a higher power the thermal heating of the target is negligible and, thus, going to a higher power would lead to some effects.

\section{Conclusion}

In this study the in-house plasma needle device has been proven to generate cold atmospheric pressure plasma that is highly efficient for an in vitro prevention of MRSA biofilm development. Under the specific conditions, complete inhibition of biofilm formation was noted even for the inoculum as high as $10^{6}$ MRSA cells. Therefore, the plasma application suggested by this study lies within the area of inanimate surface decontamination/sterilization. As far as in vivo application is concerned, it should be noted that the same plasma treatments utilized against MRSA biofilm had been previously tested for cytotoxicity on peripheral blood-derived mesenchymal stem cells and no cytotoxic effects were established [15].

We are well aware that the study provided results based upon an in vitro experimental model, and that further research is needed for practical application within the area of in vivo desinfection. In addition, further research into eradication of formed biofilms by plasmas that produce more effects on surfaces is planned, such as a micro atmospheric pressure plasma jet and an atmospheric pressure plasma jet.

\section{Acknowledgement}

This study was supported by Grants No. III41011, ON175039 and ON171037 from the Ministry of Education, Science and Technological Development of the Republic of Serbia.

\section{References}

[1] P. Cos, K. Toté, T. Horemans, L. Meas, Curr. Pharm. Des. 16, 2279 (2010)

[2] U. Römling, C. Balsalobre, J. Intern. Med. 272, 541 (2012)

[3] Z. Lj. Petrović et al., Journal of Physics: Conference Series 356, 012001 (2012)

[4] Z. Lj. Petrović et al., J. Serb. Chem. Soc. 77, 1689 (2012)

[5] M. Moreau, N. Orange, M. G. J. Feuilloley, Biotechnol. Adv. 26, 610 (2008)

[6] J. Goree, B. Liu, D. Drake, E. Stoffels, IEEE T. Plasma Sci. 34, 1317 (2006)

[7] B. Kim,et al., Food microbial. 28, 9 (2011)

[8] G. Fridman et al., Plasma Process. Polym. 5, 503 (2008)

[9] T. Maisch et al., PloS One 7, e34610 (2012)

[10] J. L. Zimmermann et al., New J. Phys. 14, 073037 (2012)

[11] M. G. Kong et al., New J. Phys. 11, 115012 (2009)

[12] M. Hee Lee et al., New J. Phys. 11, 115022 (2009)

[13] S. G. Joshi et al., Am. J. Infect. Control 38, 293 (2010)

[14] N. Puač et al., J. Phys. D Appl. Phys. 39, 3514 (2006)

[15] S. Lazović et al., New J. Phys. 12, 083037 (2010)

[16] N. Hoiby et al., Int. J. Oral. Sci. 3, 55 (2011)

[17] T. J. Hannan et al., FEMS Microbiol. Rev. 36, 616 (2012)

[18] C. J. Sanchez et al., BMC Infect. Dis. 13, 47 (2013)

[19] N. K. Archer et al., Virulence 2, 445 (2011)

[20] J. M. Mootz et al., Infect. Immun. 81, 3227 (2013)

[21] K. D. Weltmann et al., Pure Appl. Chem. 82, 1223 (2010)

[22] M. Laroussi, Plasma Process. Polym. 2, 391 (2005)

[23] M. Moisan et al., Int. J. Pharmaceut. 226, 1 (2001)

[24] U. Cvelbar, M. Mozetič, N. Hauptman, M. KlanjsekGunde, J. Appl. Phys. 106, 103303 (2009)

[25] S. Manola, Z. Lj. Petrović, R. M. Jankov, In: M. Milosavljević (Ed.), 16th SPIG XVI Summer School and International Symposium on the Physics of Ionized Gases, Serbia (Belgrade 1993) 285

[26] K. Stapelmann, O. Kylián, B. Denis, F. Rossi, J. Phys. D: Appl. Phys. 41, 192005 (2008)

[27] D. O'Connell et al., Appl. Phys. Lett. 98, 043701 
(2011)

[28] A. von Keudell et al., Plasma Process. Polym. 7, 327 (2010)

[29] N. Philip et al., IEEE T. Plasma Sci. 30, 1429 (2002)

[30] M. Y. Alkawareek et al., FEMS Immunol. Med. Microbiol. 65, 381 (2012)

[31] E. Kvam, B. Davis, F. Mondello, A. L. Garner, Antimicrob. Agents Ch. 56, 2028 (2012)

[32] R. Matthes et al., Plasma Process. Polym. 10, 161 (2013)

[33] T. Maisch et al., J. Ind. Microbiol. Biot. 39, 1367 (2012)

[34] O. G. Brakstad, K. Aasbakk, J. A. Maeland, J. Clin. Microbiol. 30, 1654 (1992)

[35] Y. Kondo et al., Antimicrob Agents Chemother 51, 264 (2007)

[36] I. Ćirković, Ph.D. thesis, University of Belgrade (Belgrade, Serbia, 2009)

[37] G. Malović, N. Puač, S. Lazović, Z. Lj. Petrović, Plasma Sources Sci. T. 19, 034014 (2010)
[38] I. E. Kieft and E. Stoffels, New J. Phys. 6, 149 (2004)

[39] E. Stoffels, J. Flikweert, W. W. Stoffels, G. M. W. Kroesen, Plasma Sources Sci. T. 11, 383 (2002)

[40] S. Stepanović et al., APMIS 115, 891 (2007)

[41] A. G. Whittaker et al., J. Hosp. Infect. 56, 37 (2004)

[42] H. Halfmann, N. Bibinov, J. Wunderlich, P. Awakowicz, J. Phys. D., Appl.Phys. 40, 4145 (2007)

[43] Y. F. Hong et al., Lett. Appl. Microbiol. 48, 33 (2009)

[44] K. Lee, K. H. Peak, W. T. Ju, Y. Lee, J. Microbiol. 44, 269 (2006)

[45] H. Yu et al., J. Appl. Microbiol. 101, 1323 (2006)

[46] M. H. Lee et al., New J. Phys. 11, 115022 (2009)

[47] J. J. Cotter et al., J. Hosp. Infect. 78, 204 (2011)

[48] A. Ghaffari, C. C. Miller, B. McMullin, A. Ghahary, Nitric oxide 14, 21 (2006)

[49] D. O. Schairer, J. S. Chouake, J. D. Nosanchuk, A. J. Friedman, Virulence 3, 271 (2012)

[50] A. Friedman et al., Virulence 2, 217 (2011)

[51] G. Han et al., PloS One 4, e7804 (2009) 